\title{
Detection of nanomechanical vibrations by dynamic force microscopy in higher cantilever eigenmodes
}

\author{
Alvaro San Paulo, ${ }^{\text {a) }}$ Justin P. Black, Richard M. White, and Jeffrey Bokor \\ Department of Electrical Engineering and Computer Sciences, University of California, Berkeley, California \\ 94720
}

(Received 25 May 2007; accepted 9 July 2007; published online 2 August 2007)

\begin{abstract}
The authors present a method based on dynamic force microscopy to characterize subnanometer-scale mechanical vibrations in resonant micro- and nanoelectromechanical systems. The method simultaneously employs the first eigenmode of the microscope cantilever for topography imaging and the second eigenmode for the detection of the resonator vibration. Here, they apply this scheme for the characterization of a $1.6 \mathrm{GHz}$ film bulk acoustic resonator, showing that it overcomes the main limitations of acoustic imaging in contact-mode atomic force microscopy. The method provides nanometer-scale lateral resolution on arbitrarily high resonant frequency systems, which makes it applicable to a wide diversity of electromechanical systems. (C) 2007 American Institute of Physics. [DOI: 10.1063/1.2767764]
\end{abstract}

Resonant micro- and nanoelectromechanical systems (MEMS and NEMS) are attracting a growing interest in various technological and basic research areas. Radio frequency (rf) mechanical resonators with very high quality factors, optimized integration capabilities, and reduced production costs promise to substitute standard integrated circuit technology for the development of wireless communication components such as filters or duplexers. ${ }^{1,2}$ On the other hand, miniaturization of mechanical resonators has enabled the investigation of basic physical phenomena such as quantum behavior of mesoscopic systems or the ultimate limit of mass sensitivity. ${ }^{3,4}$ In spite of the important advances made so far, many issues regarding the study of resonant MEMS and NEMS remain hard to address due to the difficulty of measuring very small vibrations at high resonant frequencies. Imaging methods based on atomic force microscopy (AFM) are emerging as a powerful alternative to conventional electrical or optical characterization techniques alone, as they provide otherwise inaccessible information about MEMS and NEMS such as their spring constants, mechanical resonances, mode shapes, or power dissipation mechanisms. ${ }^{5-8}$

Dynamic force microscopy (DFM) methods ${ }^{9}$ are among the most sensitive imaging tools used for the characterization of materials, biological systems, or functional devices, and they are generally chosen whenever minimum sample perturbation is required. A basic principle common to all DFM methods is the use of any of the characteristic magnitudes of the driven microscope cantilever vibration for the measurement of a specific sample property. Recently, the use of higher-order cantilever bending eigenmodes has been shown as a versatile approach to further enhance the sensitivity of DFM methods. ${ }^{10-14}$ In this work, we demonstrate the use of the second bending eigenmode of the cantilever to detect and image mechanical vibrations in resonant MEMS and NEMS with subnanometer-scale sensitivity. The proposed method is based on standard amplitude modulated DFM (AM-DFM) operation in air, ${ }^{9}$ is applicable to arbitrarily large resonant frequency values in the rf range, and is appropriate for a

${ }^{\text {a)} P r e s e n t ~ a d d r e s s: ~ I n s t i t u t o ~ d e ~ M i c r o e l e c t r o ́ n i c a ~ d e ~ B a r c e l o n a, ~ C N M-C S I C, ~}$ 08193 Bellaterra, Spain; electronic mail: alvaro.sanpaulo@cnm.es broad range of NEMS and MEMS devices. Here we describe the performance and advantages of the method through characterization of the acoustic vibrations of a $1.6 \mathrm{GHz}$ aluminum nitride (AIN) film bulk acoustic resonator (FBAR). FBAR duplexer technology is among the most extended applications of rf-MEMS for frequency control in wireless electronics. ${ }^{15}$ Our results show that AM-DFM operation overcomes several important limitations of vibration imaging in contact-mode AFM, such as slow scan speed, damping of the resonator amplitude by the tip-sample interaction force, and the undesired excitation of thermomechanical effects.

The FBAR device used in this work [Fig. 1(a)] consists of a released piezoelectric AlN pentagonal-shaped membrane (side lengths from 40 to $80 \mu \mathrm{m}$ ) sandwiched between a bottom platinum electrode and a top aluminum electrode. The structure is designed to have a main thickness-extensional mode at $1.6 \mathrm{GHz}$ in which the device undergoes out-of-plane motion with an amplitude of up to a few nanometers. The reflection coefficient $\left(S_{11}\right)$ of the FBAR indicated a series resonant frequency of $1.595 \mathrm{GHz}$. During AFM testing, the FBAR is driven at the series resonance with an input power of $0 \mathrm{dBm}$.

(a) FBAR
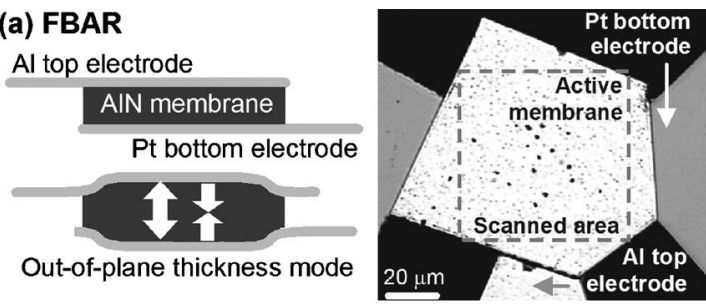

(b) AFM Setup

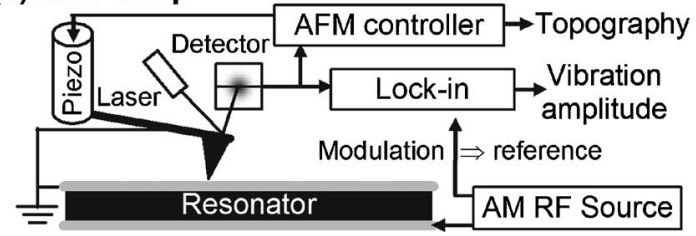

FIG. 1. Schematic representation and optical micrograph of the film bulk acoustic resonator used as test sample (a). Atomic force microscopy setup employed in this work (b). 

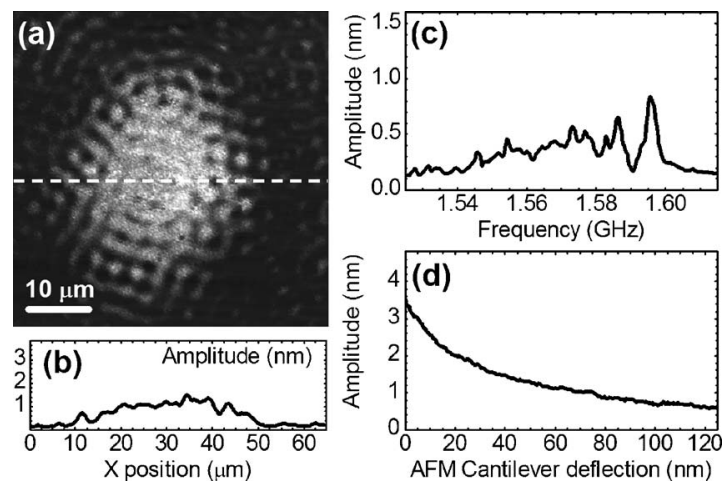

FIG. 2. FBAR characterization results obtained in contact-mode AFM: vibration amplitude image (a) and cross section (b), frequency response (c), and effect of tip-sample force (d).

A previously reported method for imaging vibrations of rf resonators is based on standard contact-mode AFM (Ref. 6) [Fig. 1(b)]. The resonator is driven with an amplitude modulated signal, where the carrier is tuned in the vicinity of the resonant frequency of the resonator and the modulation frequency $f_{\text {mod }}$ lies below the fundamental resonant frequency of the AFM cantilever (denoted here as $f_{1}$ ). As the AFM tip is scanned across the resonator surface, it follows the envelope of the resonator vibration and exhibits an amplitude equal to the rf resonator amplitude and a frequency equal to $f_{\text {mod }}$. The amplitude value can be inferred from the laser photodetector output with an external lock-in amplifier tuned to $f_{\text {mod. }}$ Probes typically used with this method are standard $\mathrm{Si}_{3} \mathrm{~N}_{4}$ cantilevers with nominal force constants around $0.1-0.5 \mathrm{~N} / \mathrm{m}$ and resonant frequencies $\left(f_{1}\right)$ that lie between 20 and $80 \mathrm{kHz}$.

Figure 2 shows the results of characterization of the FBAR with contact-mode AFM. Figures 2(a) and 2(b) are the amplitude image and cross-section profile, respectively, obtained at resonance on the $65 \times 65 \mu \mathrm{m}^{2}$ area of the resonator indicated in Fig. 1(a). The image shows the usual features observed on FBAR vibrations: the main pancakeshaped mode is recognized as a central amplitude maximum that decays toward the borders of the resonator, and a superimposed shorter wavelength pattern, corresponding to a simultaneously excited parasitic mode, is also observed. ${ }^{8}$ The mechanical frequency response displayed in Fig. 2(c) shows the main resonance peak at $1.595 \mathrm{GHz}$. Additional peaks at lower frequencies corresponding to lateral modes ${ }^{16}$ are observed with amplitudes smaller but comparable to that of the main resonant mode.

Despite providing the subnanometer-scale sensitivity, vibration imaging by contact-mode AFM has three important limitations: very large image acquisition times, perturbation of the resonator mode shape due to tip-sample interaction effects, and the possible presence of image artifacts due to thermo-mechanical effects in the resonator. The acquisition time for the amplitude image shown in Fig. 2(a) was $30 \mathrm{~min}$ (scan speed of $20 \mu \mathrm{m} / \mathrm{s}$, and $256 \times 256$ pixels). Faster scan speed resulted in significant image distortion. Image acquisition time is limited by the modulation frequency $f_{\text {mod }}$ because this sets the time constant $\tau_{\mathrm{LI}}$ of the lock-in amplifier.

The effect of tip-sample interaction is shown in Fig. 2(d), which plots the resonator amplitude as a function of the AFM cantilever set point deflection. The data were collected by measuring the resonator amplitude while a standard force Downloaded 18 Feb 2010 to 161.111.180.191. Redistribution subject
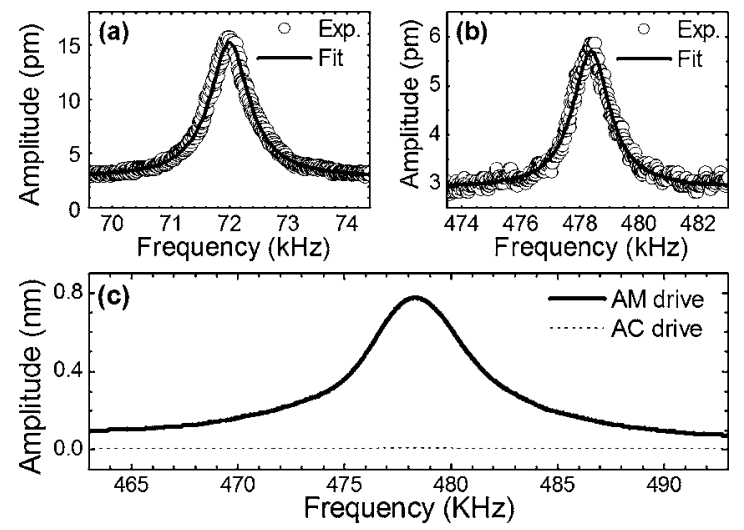

FIG. 3. Thermal spectrum of the AM-DFM cantilever around its first (a) and second (b) eigenmodes. Excitation of the second cantilever eigenmode by the FBAR vibration (c).

curve was acquired. The graph shows that the resonator amplitude decreases as the cantilever deflection, i.e., the tipsample normal force, increases. Several causes are hypothesized to contribute to this behavior. First, the tip force could shift the resonant frequency of the FBAR. Second, tipsample interaction likely introduces additional energy dissipation mechanisms (adhesion hysteresis, friction, and capillarity forces) into the system that would reduce the FBAR quality factor. A detailed analysis of these effects is beyond the scope of this letter. Regardless of its cause, tip-induced resonator amplitude variation is an effect to be avoided for an accurate characterization of the resonator.

Thermomechanical effects on AFM imaging of FBAR resonators were described in a previous work. ${ }^{17}$ Briefly, when a rf driving signal is applied to the FBAR, part of the energy is absorbed by the membrane and converted into heat. As a result, the membrane undergoes a thermal expansion proportional to the temperature. The time it takes to the membrane to heat up and reach thermal equilibrium is related to the thermal relaxation time $\tau_{\mathrm{TH}}$ of the structure, which is typically in the microsecond range. If the FBAR drive signal is modulated at a frequency $f_{\text {mod }}$ that is lower or comparable to $1 / \tau_{\mathrm{TH}}$, the membrane bows periodically at $f_{\text {mod }}$ and this introduces a background signal artifact in the vibration images. However, faster modulation frequencies decrease the magnitude of temperature fluctuations of the membrane and reduce thermally induced vibrations.

We now describe how AM-DFM overcomes these limitations while providing subnanometer-scale sensitivity for imaging mechanical vibrations. In our method, the tip is scanned in conventional AM-DFM mode, where the amplitude of the first bending eigenmode of the cantilever is used for feedback control and topography imaging. Operation in the repulsive interaction regime ${ }^{18,19}$ provided the best results in our experiments. The resonator is driven by an amplitude modulated signal with a carrier frequency at or near its resonant frequency and with a modulation frequency that matches the resonant frequency of the second AFM cantilever bending mode $\left(f_{2}\right)$. As a result, this second mode is excited due to the interaction between the tip and the vibrating surface of the resonator, and its amplitude can be measured with an external lock-in amplifier. Prior to FBAR characterization, the thermal spectrum of the free cantilever was measured with a spectrum analyzer [Figs. 3(a) and 3(b)]. The cantilever used in the experiments has a nominal spring conto AIP license or copyright; see http://apl.aip.org/apl/copyright.jsp 

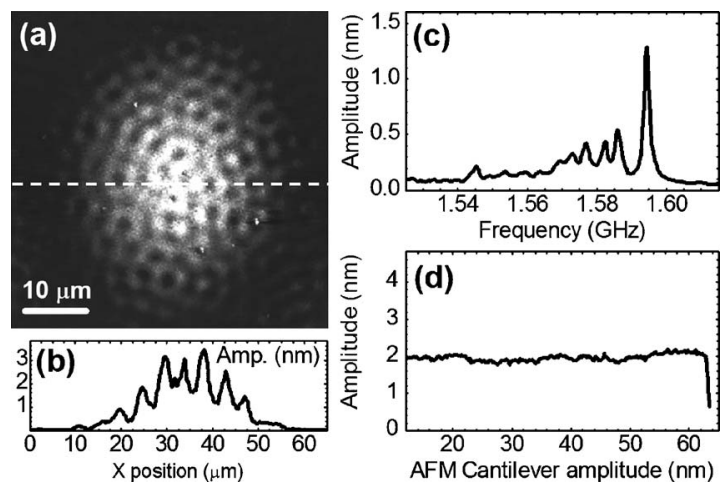

FIG. 4. FBAR characterization results obtained in AM-DFM: vibration amplitude image (a) and cross section (b), frequency response (c), and effect of tip-sample force (d).

stant of $2 \mathrm{~N} / \mathrm{m}$ and resonant frequencies of $f_{1}=72 \mathrm{kHz}$ and $f_{2}=478 \mathrm{kHz}$.

Figure 3(c) shows the effect of the FBAR vibration on the AFM tip vibration amplitude during AM-DFM operation, as obtained with the lock-in detection scheme described above. When the resonator is driven at resonance and the modulation frequency $f_{\text {mod }}$ is swept around $f_{2}$, the vibration signal reaches a maximum at $f_{\text {mod }}=f_{2}$. However, when the resonator is turned off or driven with a constant amplitude signal, the signal detected is negligible. This result indicates that the signal observed when driving the resonator with an AM signal at $f_{\text {mod }}=f_{2}$ is produced by excitation of the second AFM cantilever eigenmode. As the FBAR resonant frequency $(1.6 \mathrm{GHz})$ is much higher than $f_{1}$ and $f_{2}$ of the cantilever, the tip senses an effective position of the surface, which is given by the envelope of the FBAR vibration. With the FBAR modulated at $f_{\text {mod }}=f_{2}$, the tip is subject to quasiperiodic excitation due to its interaction with the FBAR vibration envelope, which produces the excitation of the second cantilever eigenmode.

Figure 4 shows the FBAR characterization results obtained with AM-DFM. The amplitude image and crosssection profile in Fig. 4 show the same basic features as the contact-mode results of Fig. 3. In the images of Figs. 4(a) and 3(a), the overall magnitude as well as the spatial distribution of the vibration amplitude are similar. However, remarkable improvements in brightness and contrast are clearly observed. The peaks and valleys resulting from the short wavelength parasitic modes are much more clearly resolved in AM-DFM. The frequency response of the resonator [Fig. 4(c)] also shows more sharply defined features: the main resonant peak is more clearly identified from the lateral mode peaks and it reaches a maximum amplitude of $1.3 \mathrm{~nm}$, almost twice as large as the maximum peak amplitude observed in contact mode.

We attribute these significant improvements to the minimization of tip-sample interaction forces, a well-known general feature of AM-DFM. ${ }^{9}$ This was confirmed by measuring the resonator amplitude versus the AFM cantilever set point amplitude [Fig. 4(d)]. In AM-DFM mode, the set point amplitude sets the effective tip-sample interaction force. ${ }^{19}$ In our experiments, we do not observe any dependence of the
FBAR amplitude on the set point amplitude, which implies that the force exerted in this mode has no effect on the resonator vibration.

Modulation of the FBAR at $f_{\text {mod }}=f_{2}$ has two additional advantages. First, $f_{\text {mod }}$ is now more than five times larger than the frequencies typically used in contact mode. As $f_{\text {mod }}$ limits the lock-in amplifier time constant and hence the overall image acquisition time, scan speed can now be up to five times faster. The image in Fig. 4(a) was taken in 7 min (scan speed of $80 \mu \mathrm{m} / \mathrm{s}$ and $256 \times 256$ pixels). The second advantage concerns thermomechanical effects in acoustic devices. As discussed above, faster modulation rates minimize thermally induced modes because there is less time for the device to heat up and cool down during each modulation cycle. At the modulation rate required to match the second eigenmode of the cantilever, we did not observe any thermomechanically induced artifacts in the FBAR.

In summary, we have developed an AM-DFM based method for the characterization of mechanical vibrations in MEMS and NEMS with subnanometer-scale sensitivity. The method has been demonstrated here for the characterization of a $1.6 \mathrm{GHz}$ FBAR, but it is applicable to a very wide variety of resonant devices, regardless of their resonant frequency. In particular, given that lateral resolution in DFM is limited by the tip radius (typically below $10 \mathrm{~nm}$ ), and that DFM operation ensures minimization of tip-sample forces, this method could be directly applied on much smaller NEMS, where optical methods are not applicable.

This work was supported by the National Science Foundation (Grant No. EEC-0425914).

${ }^{1}$ S. Lucyszyn, IEE Proc.: Sci., Meas. Technol. 151, 93 (2004).

${ }^{2}$ C. T. C. Nguyen, IEEE Trans. Ultrason. Ferroelectr. Freq. Control 54, 251 (2007).

${ }^{3}$ M. D. LaHaye, O. Buu, B. Camarota, and K. C. Schwab, Science 304, 74 (2004).

${ }^{4}$ Y. T. Yang, C. Callegari, X. L. Feng, K. L. Ekinci, and M. L. Roukes, Nano Lett. 6, 583 (2006).

${ }^{5}$ A. San Paulo, J. Bokor, R. T. Howe, R. He, P. Yang, D. Gao, C. Carraro, and R. Maboudian, Appl. Phys. Lett. 87, 053111 (2005).

${ }^{6}$ H. Safar, R. N. Kleiman, B. P. Barber, P. L. Gammel, J. Pastalan, H. Huggins, L. Fetter, and R. Miller, Appl. Phys. Lett. 77, 136 (2000).

${ }^{7}$ B. Ilic, S. Krylov, L. M. Bellan, and H. G. Craighead, J. Appl. Phys. 101, 044308 (2007).

${ }^{8}$ A. San Paulo, E. Quevy, J. Black, R. T. Howe, R. White, and J. Bokor, Microelectron. Eng. 84, 1354 (2007).

${ }^{9}$ R. Garcia and R. Perez, Surf. Sci. Rep. 47, 197 (2002).

${ }^{10}$ R. W. Stark, T. Drobek, and W. M. Heckl, Appl. Phys. Lett. 74, 3296 (1999).

${ }^{11}$ M. Stark, R. W. Stark, W. M. Heckl, and R. Guckenberger, Proc. Natl. Acad. Sci. U.S.A. 99, 8473 (2002).

${ }^{12}$ T. R. Rodriguez and R. Garcia, Appl. Phys. Lett. 84, 449 (2004).

${ }^{13}$ O. Sahin, C. F. Quate, O. Solgaard, and A. Atalar, Phys. Rev. B 69, 165416 (2004)

${ }^{14}$ R. Proksch, Appl. Phys. Lett. 89, 113121 (2006).

${ }^{15}$ R. Ruby, P. Bradley, J. D. Larson, and Y. Oshmyansky, Electron. Lett. 35, 794 (1999).

${ }^{16}$ K. L. Telschow, V. A. Deason, D. L. Cottle, and J. D. Larson, IEEE Trans. Ultrason. Ferroelectr. Freq. Control 50, 1279 (2003).

${ }^{17}$ A. San Paulo, X. Liu, and J. Bokor, Appl. Phys. Lett. 86, 084102 (2005).

${ }^{18}$ R. Garcia and A. San Paulo, Phys. Rev. B 60, 4961 (1999).

${ }^{19}$ A. San Paulo and R. Garcia, Biophys. J. 78, 1599 (2000). 